\title{
Review of "Exploratory Social Network Analysis with Pajek" by Wouter De Nooy, Andrej Mrvar and Vladimir Batageli
}

\author{
Muaz A. Niazi ${ }^{*}$ (D), Athanasios Vasilakos ${ }^{2}$ and Anatoly Temkin ${ }^{3}$
}

*Correspondence:
muaz.niazi@gmail.com
${ }^{1}$ COSMOSE Research
Group, Computer Science
Department, COMSATS
University, Islamabad,
Pakistan
Full list of author information
is available at the end of the
article

${ }^{*}$ Correspondence: muaz.niazi@gmail.com

' COSMOSE Research Group, Computer Science

Department, COMSATS

Pakistan

\author{
Book details \\ De Nooy W., Mrvar A., and Batagelj V. \\ Exploratory Social Network Analysis with Pajek \\ 3rd (Expanded) Edition \\ Cambridge: Cambridge University Press; 2018. \\ 334 pages, ISBN 978-1-108-56569-1
}

Keywords: Social network analysis, Complex adaptive systems, Software tool

\section{Introduction}

Human behavior can be considerably complex. The human society is self-organized in a number of different ways ranging from social groups (Koponen and Nousiainen 2016; Sharma et al. 2016) to clans, countries, nations, and societies. "Social network analysis" is a well-established field for quantitatively understanding Complex Societal behavior (Sharma et al. 2016). One application of this domain is in the discovery of complex patterns in different areas. Ideas surround this domain range from Online social networks (Mauri et al. 2011) to Complex Communication and citation networks (Khan and Niazi 2018; Niazi and Hussain 2011). Not only understanding the application of social network theory allows us to infer about central nodes, it can also allow for understanding the induction of emotions in social media users (Chirico et al. 2017).

While social network analysis is an exciting domain, arguably one of the primary reasons for its widespread usage is the wide availability of social network analysis software and datasets. Among numerous other tools, "Pajek" (Mrvar and Batagelj 2016) is one of the key freely available, professional class social network analysis tools. Among its various strengths is its ability to handle up to 10 billion vertices-capability introduced recently. The book under review has been written primarily for this fundamentally redesigned version of Pajek. 


\section{Review}

Overall, a quick look at the book reveals that the authors have indeed presented an unparalleled review of the Pajek tool. It is also obvious that the book is oriented to address the needs of a broader audience interested in network science. The book is comprised of five parts. Part 1 contains two chapters covering the basic concepts of social network analysis. The next 3 parts with chapters 3-11 focus on the major applications of social network analysis in the Social Sciences using Pajek. The final part with chapters 12-13 focuses on dense and small networks. Next, we focus on the book chapters on a one by one basis.

The book commences with the first chapter explaining the basics of social network analysis (SNA) besides introducing the Pajek menu structure along with some useful network visualization commands. The second chapter presents techniques combining relational and non-relational data and attributes.

In the second part, chapter 3 elaborates on numerous techniques for cohesive subgroups based on density and connectedness including ideas covering components, $\mathrm{k}$-cores, and cliques or complete subnetworks. Chapter 4 elaborates the idea of relations and terms of affection explaining positive and negative ties. It also discusses the use of so-called balanced theory in network analysis. Chapter 5 focuses on affiliation relationships and multimodal networks besides demonstrating how 1-mode networks can be extracted from these. Finally, the chapter also introduces 3D displays.

Chapter 6 presents legacy SNA concepts such as centrality, and centralization. It also explains uni-directional communication networks in the same context. Chapter 7 discusses bridges and brokerage on a complete network and individual level respectively. It also describes holes and information flow vulnerability in networks with bridges and cut vertices and their relation to disconnectivity. Chapter 8 presents network contagion by using a contact approach. It describes how network modeling can be performed for diffusion as a contagion process. It also describes the role of personal contacts of adopters in the diffusion of innovations. Chapter 9 deals with asymmetric networks presenting structural prestige as a reception of direct and indirect positive choices. Chapter 10 describes techniques for the extraction of informal or latent ranking in directed networks. This involves the use of occurrences of different types of traits and acyclic components.

Chapter 11 covers genealogical and citation networks with a focus on methods for coping with temporal dynamics in SNA.

The last part of the book starts with Chapter 12 which moves from a description of complete exploratory structure to modeling. This chapter focuses on the so-called block models which use matrices as computational tools. Matrices are utilized as a means of social network representation.

The final chapter of the book traces randomness in SNA focusing on random graph models. These models explain how network properties and effects result from social processes rather than randomness.

The book offers an excellent overview of the Pajek tool for social network analysis. Considering that the authors of the book are also linked with the codebase and the inventors of the tool itself, this is an especially useful and much-needed volume. It is a good-read and is highly recommended for its intended readers. However, it is important 
to note here that this book does not serve and is not even designed to serve as a generic introduction to the field of social network analysis. In other words, it is focused on the Pajek tool and serves its purpose well.

\section{Abbreviation}

SNA: social network analysis.

Acknowledgements

Not applicable.

\section{Authors' contributions}

MAN conceived the idea. MAN, AV, AT were involved in writing and editing. All authors read and approved the final manuscript.

Funding

Not applicable.

Availability of data and materials

Not applicable.

Competing interests

The authors declare that they have no competing interests.

\section{Author details}

${ }^{1}$ COSMOSE Research Group, Computer Science Department, COMSATS University, Islamabad, Pakistan. ${ }^{2}$ Department of Computer Science, Electrical and Space Engineering, Lulea University of Technology, Luleå, Sweden. ${ }^{3}$ Department of Computer Science, Boston University Metropolitan College, Boston, MA, USA.

Received: 18 May 2019 Accepted: 3 June 2019

Published online: 17 June 2019

\section{References}

Chirico A, Cipresso P, Yaden DB, Biassoni F, Riva G, Gaggioli A (2017) Effectiveness of immersive videos in inducing awe: an experimental study. Sci Rep 7(1):1218

Khan BS, Niazi MA (2018) Emerging topics in internet technology: a complex networks approach. Internet Technol Lett 1(4):e41

Koponen IT, Nousiainen M (2016) Formation of reciprocal appreciation patterns in small groups: an agent-based model. Complex Adapt Syst Model 4(1):24

Mauri M, Cipresso P, Balgera A, Villamira M, Riva G (2011) Why is Facebook so successful? Psychophysiological measures describe a core flow state while using Facebook. Cyberpsychol Behav Soc Netw 14(12):723-731

Mrvar A, Batagelj V (2016) Analysis and visualization of large networks with program package Pajek. Complex Adapt Syst Model 4(1):6

Niazi M, Hussain A (2011) Agent-based computing from multi-agent systems to agent-based models: a visual survey. Scientometrics 89(2):479

Sharma I, Chourasia B, Bhatia A, Goyal R (2016) On the role of evangelism in consensus formation: a simulation approach. Complex Adapt Syst Model 4(1):16. https://doi.org/10.1186/s40294-016-0029-4

\section{Publisher's Note}

Springer Nature remains neutral with regard to jurisdictional claims in published maps and institutional affiliations. 